\title{
Shorter addition chain for smooth integers using decomposition method.
}

\begin{abstract}
An efficient computation of scalar multiplication in elliptic curve cryptography can be achieved by reducing the original problem into a chain of additions and doublings. Finding the shortest addition chain is an NP-problem. To produce the nearest possible shortest chain, various methods were introduced and most of them depends on the representation of a positive integer $\mathrm{n}$ into a binary form. Our method works out the given $\mathrm{n}$ by twice decomposition, first into its prime powers and second, for each prime into a series of 2 's from which a set of rules based on addition and doubling is defined. Since prime factorization is computationally a hard problem, this method is only suitable for smooth integers. As an alternative, the need to decompose $n$ can be avoided by choosing $n$ of the form $p 1$ e1p 2 e $2 \cdots r$ er. This shall not compromise the security of ECC since its does not depend on prime factorization problem. The result shows a significant improvement over existing methods especially when $n$ grows very large.
\end{abstract}

Keyword: Elliptic curves cryptography; Addition chain; Scalar multiplication; Binary method; Non-adjacent form; Complementary recoding. 